\title{
Dissecção e Rotura da Artéria Pulmonar Associada a Persistência do Canal Arterial: Relato de um caso
}

\author{
Mabel de Moura Barros ZAMORANO*
}

RBCCV 44205-34

\begin{abstract}
ZAMORANO, M. M. B. - Dissecção e rotura da artéria pulmonar associada a persistência do canal arterial: relato de um caso. Rev. Bras. Cir. Cardiovasc., 2 (2): 139-144, 1987.

RESUMO: É descrito um caso de dissecção da artéria pulmonar, com rotura intrapericárdica e tamponamento cardiaco, em menina de 13 anos de idade, portadora de canal arterial grande e valva pulmonar bicúspide. Havia hipertensão pulmonar com arteriopatia plexogênica grave: nas grandes artérias elásticas. a camada média tinha alteraçōes necróticas e reparadoras profundas. Discute-se o papel da medionecrose, condiçâo que é exacerbada pela hipertensão e por caracteristicas individuais, na patogenia do aneurisma dissecante e no caso em questão. A análise da literatura mostra que, na artéria pulmonar, a dissecção é sempre um acidente fatal, por rotura ou obstrução arterial. $O$ tratamento desta intercorrência, eminentemente cirúrgico, raramente é possivel, devido ao caráter fulminante da evolução e à ausência de sinais definidos para o diagnóstico em tempo hábil.
\end{abstract}

DESCRITORES: Artéria pulmonar, dissecção; persistência de canal arterial.

\section{INTRODUÇÃO}

A dissecção arterial pulmonar é, sempre, relacionada à hipertensão arterial deste território, como uma intercorrência rara. Em muitos dos trabalhos disponiveis, os casos de dissecção são relatados juntamente com outros tipos de rotura da artéria pulmonar, o que dificulta o estabelecimento da real incidência desta complicação. Em 1934, FAVORITE ${ }^{10}$ conseguiu reunir 8 casos de rotura da artéria pulmonare acrescentou mais um, porém destes 9, apenas o de Duffield se refere, seguramente, a rotura arterial por dissecção. Um dos trabalhos, em que se teve o cuidado de separar a rotura por dissecção daquelas de outro tipo, é o de LEVY ${ }^{20}$, publicado em 1961 , onde se coletam mais 14 casos de aneurisma dissecante pulmonar. Numa revisão posterior, SHILKIN et alii ${ }^{27}$ adicionaram 9 casos e, em 1981, mais 7 relatos foram alinhados por LUCHTRATH ${ }^{21}$. Percorrendo a literatủra médica, conseguimos detectar mais 12 comunicaçōes, totalizando, então, 43 casos de dissecçāo pulmonar inqüestionável ${ }^{10.23}$, que, certamente, nāo representam o número total de descrições, por não incluírem trabalhos em idiomas como o russo ou o japonês. Nesta amostra, é quase constante a concomitância com hipertensão pulmonar, embora de etiologia variada; as únicas exceçōes tratam de 1 caso em que a dissecção se deveu a arterite por células gigantes ${ }^{5}$ e outro, citado por SHIL$\mathrm{KIN}$ et alii ${ }^{27}$, em que a única patologia referida é a agressão luética da artéria pulmonar.

Os aneurismas dissecantes pulmonares foram descritos em associação com hipertensão pulmonar primária $^{21,27}$, doença pulmonar obstrutiva crônica ${ }^{2,27}$, estenose mitral ${ }^{20,27,32}$, arterites $^{8,20,32}$, tuberculose ${ }^{11,20 .}$ ${ }^{27}$, esquistossomose ${ }^{22}$, ou como conseqüência de traumas arteriais por catéter de Swan-Ganz ${ }^{13}{ }^{26}$. Porém, em 19 dos casos levantados, a dissecção pulmonar complicou hipertensão induzida por cardiopatia congênita, estando a persistência do canal arterial presente em 15 destes (Tabela 1). Os 4 casos restantes se referem a malformaçōes outras, como comunicaçāo interatrial (caso de Laubry, citado por DETTERLING Jr. \& CLAGETT ${ }^{9}$ ), truncus ${ }^{6}$ e comunicação interventricular ${ }^{23}$. Uma interessante associação foi relatada, em 1960, (citação

\footnotetext{
Trabalho realizado no Instituto Dante Pazzanese de Cardiologia. São Paulo, SP, Brasil.

- Do Instituto Dante Pazzanese de Cardiologia.

Entregue para publicaçăo em 31 de julho de 1987

Endereço para separatas: Mabel Barros Zamorano. Caixa Postal 215. São Paulo, SP, Brasil.
} 
ZAMORANO, M. M. B. - Dissecção e rotura da arteria pulmonar associada a persistència do canal arterial: relato de um caso. Rev Bras. Cir. Cardiovasc. 2(2):139-144, 1987.

TABELA 1

ANEURISMAS DISSECANTES PULMONARES ASSOCIADOS A PERSISTENCIA DO CANAL ARTERIAL

\begin{tabular}{|c|c|c|c|c|c|}
\hline \multirow{2}{*}{\multicolumn{2}{|c|}{$\begin{array}{l}\text { Autor } \\
01 \text { - DURNO \& BROWN, } 1908 \\
{\text { (Cit. Levy) })^{2}}^{2}\end{array}$}} & \multicolumn{2}{|c|}{ Idade/Sexo } & \multirow{2}{*}{$\begin{array}{l}\text { Causa de morte } \\
\text { Hemopericárdio }\end{array}$} & \multirow{2}{*}{$\begin{array}{c}\text { Lesão associada e } \\
-\end{array}$} \\
\hline & & $33 a$ & $M$ & & \\
\hline 02 & $\begin{array}{l}\text { - OHELA, } 1954 \\
\text { (Cit. Levy) }^{2}\end{array}$ & $31 \mathrm{a}$ & $M$ & - & - \\
\hline 03 & - WHITAKER, $1955^{12}$ & $22 a$ & $F$ & Hemopericárdio & Estenose mitral \\
\hline & $\begin{array}{l}\text { - FLEMING, } 1956 \\
\text { (Cit. Levy) }^{2}\end{array}$ & $26 a$ & M & Hemopericárdio & - \\
\hline 05 & $\begin{array}{l}\text { - GRINVAL'D, } 1959 \\
\text { (Cit. Shilkin) }^{3}\end{array}$ & $41 \mathrm{a}$ & $\mathrm{F}$ & Hemopericárdio & - \\
\hline & - FOORD \& LEWIS, $1959^{8}$ & $26 a$ & $\mathrm{~F}$ & Hemorragia mediastinal & - \\
\hline & - RAVINES, $1960^{13}$ & $36 a$ & $\mathrm{~F}$ & Hemorragia pulmonar & $\begin{array}{l}\text { 38: PO de cirurgia do canal; } \\
\text { dissecção intrapulmonar }\end{array}$ \\
\hline & $\begin{aligned}- & \text { KAPANCI, } 1965 \\
& \left.{ }^{(C i t . ~ S h i l k i n ~}\right)^{3}\end{aligned}$ & $26 d$ & M & $\begin{array}{l}\text { Obstrução arterial sem ro- } \\
\text { tura }\end{array}$ & - \\
\hline 09 & - BEST, $1967^{14}$ & $24 a$ & $\mathrm{~F}$ & Hemopericárdio & Coarctaçẫo aórtica \\
\hline 10 & - SHULL, $1970^{15}$ & $15 a$ & $\mathrm{~F}$ & Hemopericárdio & Comunicação atrial \\
\hline & - D'ARBELA, $1970^{16}$ & $18 a$ & $\mathrm{~F}$ & Hemopericárdio & $\begin{array}{l}\text { Estenose pulmonar. Parto } \\
\text { normal há } 4 \text { dias }\end{array}$ \\
\hline 12 & $\begin{aligned}- & \text { TIKOFF, } 1970 \\
& \text { (Cit. Luchtrath) }^{4}\end{aligned}$ & $23 a$ & $M$ & Hemopericárdio & Comunicação ventricular \\
\hline 13 & - COLEMAN, $1980^{17}$ & $14 a$ & $M$ & Hemopericárdio & - \\
\hline 14 & - GILLAN, $1984^{18}$ & $02 a$ & $\mathrm{~F}$ & Hemorragia mediastinal & $\begin{array}{l}\text { S. Marfan: dissecção no } \\
\text { ducto }\end{array}$ \\
\hline 15 & - HANKINS, $1985^{19}$ & $33 a$ & $\mathrm{~F}$ & Hemopericárdio & Parto normal há 17 horas \\
\hline 16 & - NOSSO CASO & $13 a$ & $\mathrm{~F}$ & Hemopericárdio & Valva pulmonar bicúspide \\
\hline
\end{tabular}

de LUCHTRATH ${ }^{21}$ ), onde a dissecção ocorreu em portador de tetralogia de Fallot com valva pulmonar atrésica, que havia sido submetido a cirurgia de Blalock 13 anos antes do óbito.

A observação que ora relatamos é mais uma associação de dissecção pulmonar com persistência do canal arterial e tem como característica interessante sua ocorrência precoce, aos 13 anos de idade. Nosso caso se assemelha ao de COLEMAN et alii $^{5}$, onde năo havia outras comunicações intracardiacas, ou síndrome de Marfan.

\section{RELATO DO CASO}

Paciente do sexo feminino, com 13 anos de idade, de raça amarela. Procurou o setor de triagem do Instituto Dante Pazzanese de Cardiologia, referindo dispnéia e cansaço desde o nascimento. A partir dos 7 anos de idade, evoluíra com cianose labial e de extremidades e episódios gripais freqüentes. Não tinha história familiar positiva para cardiopatias. Ao exame, observou-se criança hipodesenvolvida, com dedos hipocráticos, cianose perilabial e de extremidades e estase jugular. Precórdio abaulado, palpando-se 2 a. bulha de grande intensidade e constantemente desdobrada. Ictus não palpável. $\mathrm{Na}$ ausculta, havia hiperfonese e desdobramento de $2 \mathrm{a}$. buIha, com componente pulmonar aumentado; sopro sistólico proto-meso ejetivo na área pulmonar, irradiado para o lado esquerdo do pescoço. O restante do exame físico era normal. O eletrocardiograma (ECG) mostrava sobrecarga ventricular direita e, na radiografia de tórax, chamava a atenção grande abaulamento da artéria pulmonar e fechamento da vasculatura na periferia dos campos pulmonares. No retorno seguinte, enquanto aguardava atendimento, a paciente apresentou crise súbita de cianose, sendo levada à emergência, onde se constatou parada cardiaca, que não reverteu com as manobras usuais de ressuscitação cardiopulmonar.

Ao exame necroscópico, observou-se criança hipodesenvolvida, sem estigmas de síndrome de Marfan, com alterações relevantes restritas ao sistema cardiovascular e aos pulmões. Já na abertura do tórax, via-se grande distenção hemorrágica do saco pericárdico. 0 tronco pulmonar, com localização normal, era muito aumentado e tinha serosa hemorrágica, onde se via ponto de soluçāo de continuidade, logo acima do anel valvar. A abertura desta artéria mostrou dissecçăo de sua parede e extensa laceração intimal (Figura 1), que ia da região supravalvar até a emergência de um canal arterial com $1,0 \mathrm{~cm}$ de diâmetro interno e $1,2 \mathrm{~cm}$ de comprimento. No restante do tronco pulmonar, a superfície interna era 
ZAMORANO, M. M. B. - Dissecção e rotura da artéria pulmonar associada a persistência do canal arterial: relato de um caso. Rev. Bras. Cir. Cardiovasc. 2(2):139-144, 1987

irregular, rugosa, com pequenas placas esbranquiçadas e endurecidas. $\mathrm{O}$ exame microscópico mostrou extenso hematoma na camada média, que se dirigia para a adventícia; variados graus de espessamento fibroso da intima estavam presentes, ao lado de importante degeneração da camada média, sob a forma de fragmentação e perda das lâminas elásticas, com ampla substituição colágena e cistos mucóides (Figura 2). O coração tinha câmaras normalmente postas, sem comunicações anômalas; o ventrículo direito era hipertrofiado e a valva pulmonar, bicúspide, mas não estenótica. A aorta ascendente, embora macroscopicamente normal, tinha fibrose intimal importante e rotura e perda de lâminas elásticas em suas porções mais internas, porém sem degeneração cistica. Os pulmões mostravam vasculatura saliente na superfićie de corte, notando-se placas amareladas na intima das artérias maiores. O exame microscópico caracterizou quadro hipertensivo grave, com artérias de média e intima concentricamente espessadas (Figura 3). Havia, também, pontos de necrose na parede arterial (Figura 4), bem como formação de lesōes plexiformes e algumas lesōes dilatadas (Figura 5). As artérias maiores exibiam camada média com lâminas elásticas escassas e fragmentadas, substituídas por fibrose; a intima, nestes vasos, tinha fibrose e aterosclerose.

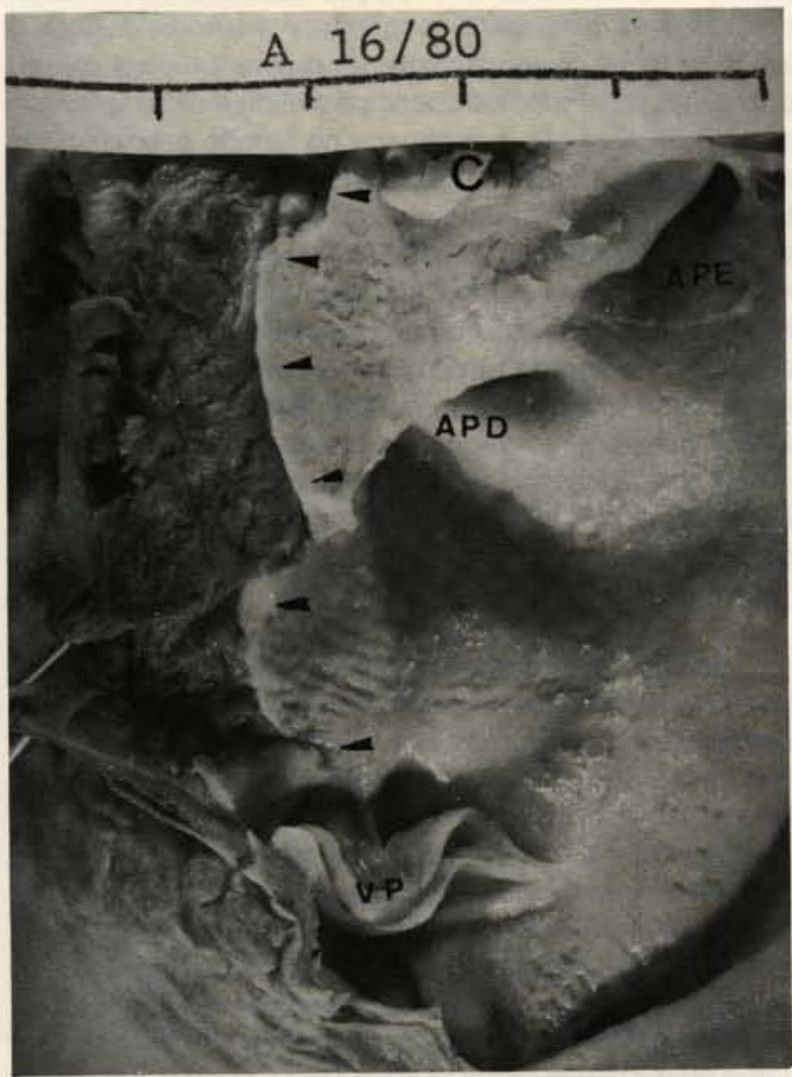

Fig. 1 - Artéria pulmonar aberta, mostrando a rotura intimal (setas) que se inicia logo acima da valva e vai até a emergência do cana arterial $(\mathrm{C})$; VP = valva pulmonar bicúspide; $\mathrm{APE}=$ artéria pulmonar esquerda; $\mathrm{APD}=$ artéria pulmonar direita.

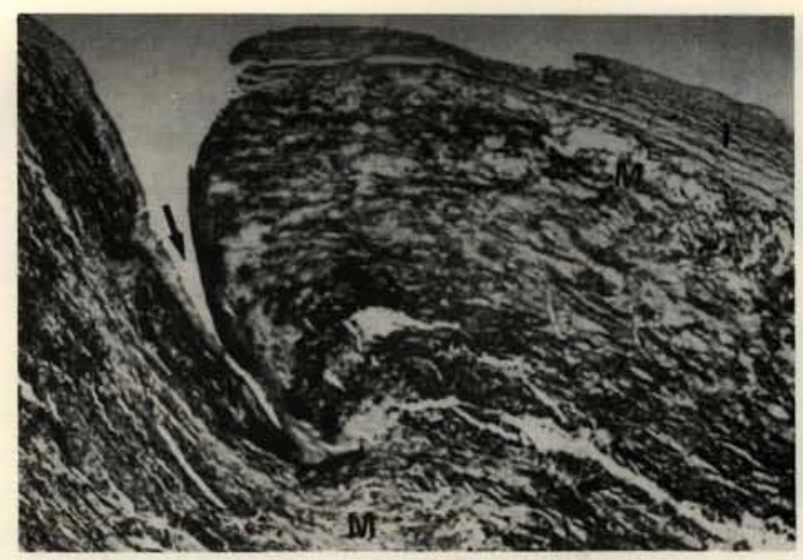

Fig. 2-Parede da artéria pulmonar na zona de rotura (seta). Íntima (1) espessada por fibrose. Camada média (M) com lâminas elásticas fragmentadas, fibrose e degeneração cistica. (Coloração para fibras elásticas $160 \mathrm{X}$ ).

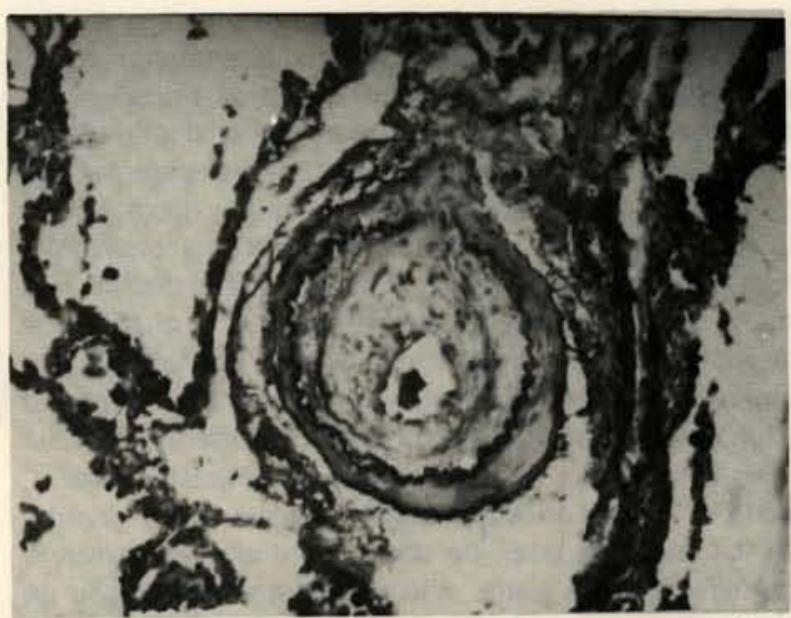

Fig. 3 - Artéria muscular intrapulmonar com espessamento fibroso concêntrico da camada intima. (Coloração para fibras elásticas $160 \mathrm{X}$ ).

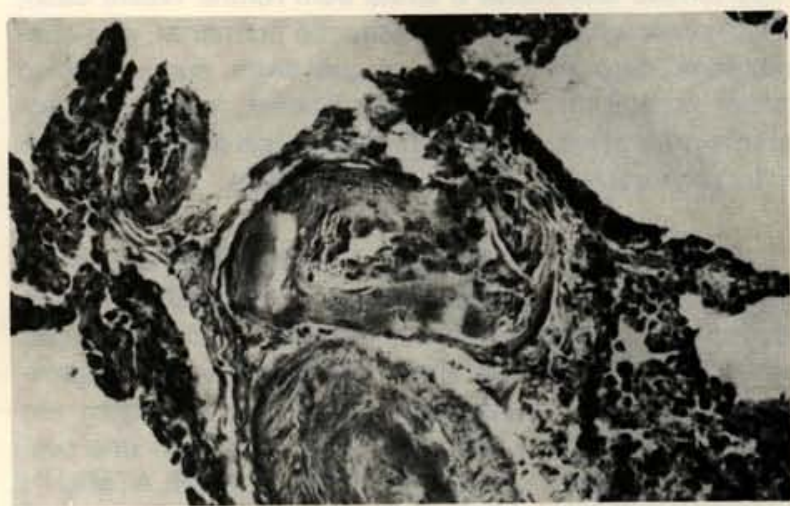

Fig. 4-Artéria intrapulmonar com necrose e fragmentação da parede (hematoxilina-eosina $160 \mathrm{X}$ ) 
ZAMORANO, M. M. B. - Dissecção e rotura da artéria pulmonar associada a persistência do canal arterial: relato de um caso. Rev. Bras. Cir. Cardiovasc. 2(2):139-144, 1987

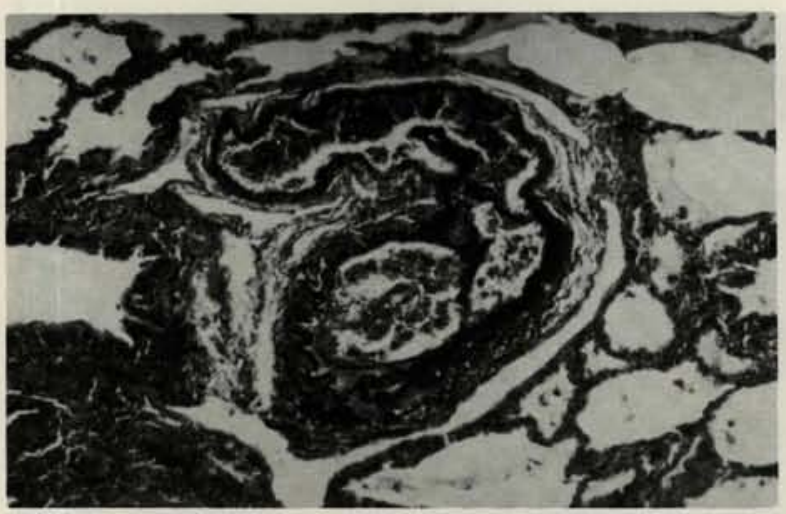

Fig. 5 - Artéria intrapulmonar com adelgaçamento segmentar da parede e formação de lesảo dilatada. (Coloração para fibras elásticas $60 \mathrm{X}$ ).

\section{COMENTÁRIOS}

A associação de dissecção da artéria pulmonar com hipertensão, na imensa maioria dos casos descritos na literatura, termina em rotura, que é diretamente responsável pelo óbito. Em apenas dois trabalhos, encontramos dissecção pulmonar em rotura, mesmo com hipertensão: um trata de paciente com canal arterial persistente, que morreu por obstrução da artéria pulmonar dissecada ${ }^{27}$. O outro relata portador de hipertensão esquistossomótica, que faleceu por insuficiência cardíaca direita ${ }^{22}$. A rotura do aneurisma dissecante resulta em quadros de tamponamento cardíaco, hemotórax ou hemorragia pulmonar, que, uma vez instalados, seguem curso rápido e fulminante. Raramente, o processo de dissecção evolui mais lentamente, como no relato de SHILKIN et alii ${ }^{27}$, onde os sinais de organização indicam que o quadro se iniciou dias antes do óbito. Contudo, ao contrário da dissecção na aorta, não encontramos definição de sinais clínicos ou radiológicos que permitam intervençâo curativa ou preventiva, no aneurisma dissecante pulmonar. O único caso onde se descreve tratamento cirúrgico, com sucesso, da dissecção pulmonar é o de DENNISON et $a / i i^{8}$, que trata de lesão induzida por arterite, sem hipertensão associada e ainda sem rotura. Neste caso, havia pontos múltiplos de dissecção pulmonar, não suspeitados até $o$ ato cirúrgico, o qual tinha, como objetivo inicial, corrigir uma regurgitação aórtica; a troca do segmento arterial lesado, utilizando um tubo de Dacron, permitiu uma evolução favorável por 3 anos.

Durante várias décadas, a etiologia da dissecção arterial foi atribuída à medionecrose ${ }^{1,30}$, juntamente com lesões outras, como necrose isquêmica, fragmentação elástica e fibrose. Estas alteraçōes têm, hoje, seu significado patogênico qüestionado, uma vez que surgem, em aortas de indivíduos normais, como resultado dos processos naturais de envelhecimento ${ }^{28}$. SCHLATMANN \& BECKER ${ }^{29}$ afirmam que estas alterações são o resultado da ação de forças hemodinâmicas sobre a parede arterial, ao longo dos anos, vindo a se tornar mais intensas e precoces nos individuos portadores de um defeito congênito qualquer que altere os componentes mediais, como na síndrome de Marfan. Tais alteraçōes também são intensificadas quando há elevação da pressāo arterial ${ }^{4,14}, 25$, ou do diâmetro do vaso, condiçōes que exacerbam a tensão sobre a parede vascular, segundo a lei de Laplace. Apesar dos estudos de SCHLATMANN \& BECKER ${ }^{28}$ e de KLIMA et alii ${ }^{19}$, onde não se demonstrou relação entre as cifras tensionais e as lesōes da média aórtica, a hipertensão arterial é um achado freqüente, nos casos de dissecção dessa artéria, confirmado em cerca de $70 \%$ dos pacientes e suspeitado em $90 \%$ das necrópsias ${ }^{25}$. Além disto, há uma relação bem evidente entre a gravidade da hipertensão e a freqüência da dissecção, fator não considerado nos trabalhos daqueles autores.

No território pulmonar, a dissecção, quase exclusivamente, coexiste com hipertensāo arterial, como já ressaltamos. E a hipertensāo leva a um exagero da necrose cística na parede da artéria pulmonar, mesmo em ausência de formaçăo aneurismática, ou de dissecçāo ${ }^{20}, 33$. TREDAL et alii ${ }^{33}$ estabeleceram uma relaçāo direta entre as lesōes mediais e o tempo de evolução e a idade dos pacientes, nos portadores de hipertensão pulmonar por cardiopatia adquirida, relação esta não confirmada nos casos de cardiopatia congênita. A ausência de correlação entre a dissecção arterial pulmonar e os niveis pressóricos, o tempo de doença e a etiologia da hipertensão fazem com que permaneça no campo especulativo a razão porque apenas alguns, entre os muitos pacientes hipertensos, tenham uma evoluçāo tão drástica. $\mathrm{Na}$ aorta, SCHLATMANN \& BECKER ${ }^{29}$ sugerem que o estimulo lesivo sobre a parede arterial leva a uma exacerbação dos fenômenos normais de injúria e reparo, propiciando a criaçāo de um círculo vicioso onde, cada vez mais, estes fenômenos se multiplicam: a dissecção, em ausência de doença intrínseca na composiçāo da parede arterial, seria apenas um acidente no curso de um processo comum. O fator individual de hiper-reatividade vascular ${ }^{15}$ talvez selecione os casos susceptiveis a este "acidente".

No caso que relatamos, não se evidenciaram sinais que sugerissem algum tipo de erro congênito na composição arterial, apesar de que, nas porções internas da aorta ascendente, encontraram-se necrose isquêmica e fibrose em intensidade incomum para a faixa etária da paciente. Havia uma doença plexogênica grave nos pulmões e a comunicação entre as duas circulaçōes era de grande magnitude. Em casos como este, espera-se que a hipertensão pulmonar esteja presente desde o nascimento e que as artérias elásticas evoluam com um padrão fetal ou "configuração aórtica" 17, 18. Contudo, o que observamos nesta criança foram artérias elásticas pulmonares com padrão "adulto" de camada média, indicando que o estado hipertensivo se instalou após a regressão fisiológica desses vasos. Embora a elevação 
ZAMORANO, M. M. B. - Dissecção e rotura da artéria pulmonar associada a persistência do canal arterial relato de um caso. Rev. Bras. Cir. Cardiovasc.. 2(2):139-144, 1987

dos niveis pressóricos não pareça ter estado presente desde o nascimento, a doença vascular teve um curso rápido e grave. A explicação patogênica da susceptibilidade desta criança é difícil, com base apenas nas suas características de hiperreatividade vascular, a me- nos que este possa ser um estado adquirido ou desencadeado tardiamente, já que houve um periodo inicial de tolerância, onde as artérias elásticas pulmoniares puderam ter a regressāo fisiológica que ocorre nos indiviciuos normais.

RBCCV $44205-34$

ZAMORANO, M. M. B. - Dissection and rupture of pulmonary artery associated to patent ductus arteriosus case report. Rev. Bras. Cir. Cardiovasc., 2 (2): 139-144, 1987.

ABSTRACT: The author presents a case of a 13-year old girl with pulmonary trunk dissection and rupture with a cardiac tamponade. There was a ductus arteriosus persistent an $\dot{z}$ a non stenotic bicuspid pulmonary valve. A plexogenic pulmonary arteriopathy and severe process of injury and repair in the elastic vessels are present. The significance of medionecrosis in the pathogenesis ct pulmonary dissection is discussed. An analysis of reports in dissecting pulmonary aneurysm reveals a faial outcome in the vast majority of them, due either to arterial rupture or obstruction.

DESCRIPTORS: pulmonary artery, dissection; patent ductus arteriosus.

\section{REFERÊNCIAS BIBLIOGRÁFICAS}

1 ALMEIDA, J. A. P. - Aneurismas de aorta e seus ramos ênfase especial à medionecrose cística idiopática. Arq. Bras. Cardiol., 25 (3): 211-219, 1972.

2 BERNHEIM, J. \& GRIFFEL, B. - A propos d'un cas d'anévrisme disséquant de l'artère pulmonaire. Ann. Anat. Pathol. (Paris), 17 (1): 83-90, 1972.

3 BEST, J. - Dissecting aneurysm of the pulmonary artery - with multiple cardiovascular abnormalities and pulmonary hypertension. Med. J. Aust., 2 (25): 1129-1130, 1967.

4 CARLSON, R. G.; LILLEHEI, C. W.; EDWARDS, J. E. Cystic medial necrosis of the ascending aorta in relation to age and hypertension. Am. J. Cardiol., 25 (4): $411-415,1970$

5 COLEMAN, M.; SLATER, D.: BELL, R, - Rupture of pulmonary artery aneurysm associated with persistent ductus arteriosus. Br. Heart J.; 44 (4): 464-468, 1980.

6 CRUMPTON, M. - Congenital heart disease and dissecting aneurysm of pulmonary artery. Br. Med. J., 1: $1303,1950$.

7 D'ARBELA, P. G.; MUGERWA, J. W.; PATEL, A. K.; SOMERS, K. - Aneurysm of pulmonary artery with persistent ductus arteriosus and pulmonar infundibular stenosis fatal dissection and rupture in pregnancy. $\mathrm{Br}$. Heart J., 32 (1) 124-126, 1970.

8 DENNISON, A. R.; WATKINS, R. M.; GUNNING, A. J. - Simultaneous aortic and pulmonary artery aneurysm due to giant cell arteritis. Thorax, 40 (2): 156-157, 1985.

9 DETTERLING Jr., R. A. \& CLAGETT, O. T. - Aneurysm of pulmonary artery: review of the literature and report of a case. Am. Heart J., 34 (1): 471-499, 1947.
10 FAVORITE, G. O. - Cor biatriatum triloculare with rudiinentary right ventricle, hypoplasia of transposed aorta and patent dictus arteriosus, terminating by rupture of dilated pulmonary artery. Am. J. Med. Sci., 187 (5): 653-671, 1934

11 FOORD, A. G. \& LEWIS, R. D. - Primary dissecting aneurysm of peripheral and pulmonary arteries: dissecting hemorrhage of media. Arch. Pathol., 68: 553-577. 1959.

12 GILLAN, J. E.; COSTIGAN, D. C.; KEELEY, F. M.; ROSE, V. - Spontaneous dissecting aneurysm of the ductus arteriosus in an infant with Marfan syndrome. J. Pediatrics, 105 (6): 952-955, 1984

13 GOMEZ-ARNAU, J.; MONTERO, C. G.; LUENGO, C.; GILSANZ, F. J.; AVELLO, F. - Pulmonary dissection and rupture of pulmonary artery after catheter use in pulmonary hypertension. Critical Care Med., 10 (10): 694-695, 1982.

14 GORE, I. \& HIRST Jr., A. E. - Dissecting aneurysm of the aorta. Progr. Cardiovasc. Dis., 16 (2): 103-111. 1973.

15 GROVER, R. F.; VOGE, J. H. K.; AVERILL, K. H.; BLOUNT, S. G. - Pulmonary hypertension: individual and species variability relative to vascular reactivity. Am. Heart j. 66 (1): 1-3, 1963.

16 HANKINS, G. D.; BREKKEN, A. L.; DAVIS, L. M. - Maternal death secondary to a dissecting aneurysm of the pulmonary artery. Obstet. Gynecol., 65 (Supl. 3): 45-48, 1985

17 HEATH, D.: DUSHANE, J. W.; WOOD, E. H.; EDWARDS, J. E. - The structure of the pulmonary trunk at diferent ages and in cases of pulmonary hypertension and pulmonary stenosis. J. Pathol. Bacteriol. 77 (2): 443-456, 1959. 
ZAMORANO, M. M. B. - Dissecção e rotura da artéria pulmonar associada a persistència do canal arterial: relato de um caso. Rev. Bras. Cir. Cardiovasc., 2(2):139-144, 1987.

18 HEATH, D. \& EDWARDS, J. E. - Configuration of elastic tissue of pulmonary trunk in idiopathic pulmonary hypertension. Circulation, 21 (1): 59-62, 1960.

19 KLIMA, T.; COELHO, A.; GRAY, A. G.; WUKASCH, D. C.; REUL Jr., G. J.; COOLEY, D. A. - The morphology of ascending aortic aneurysms. Hum. Pathol., 14 (9): $810-817,1983$

20 LEVY, H. - Partial rupture of pulmonary artery with lesions of medionecrosis in a case of mitral stenosis. Am. Heart J., 62 (1): 31-42, 1961

21 LUCHTRATH, H. - Dissecting aneurysm of the pulmonary artery, Virchows. Arch. (Pathol. Anat.), 391: 241-247. 1981.

22 MIZIARA, H. L.; FILOMENO, A. P.; YUNES, M. A. F. Aneurisma dissecante de artéria pulmonar associado a esquistossomose pulmonar: relato de um caso. Arq. Bras. Cardiol., 37 (2): 107-110, 1981.

23 PLACIK, B.; RODBARD, S.; MCMAHON, J.; SWAROOP, S. - Pulmonary artery dissection and rupture in Eisenmenger's syndrome. Vasc. Surg., 10 (2): 72-80, 1976.

24 RAVINES, H. T. - Dissecting hematomas of intrapulmonary arteries in a case of pulmonary hypertension associated with patent ductus arteriosus. J. Thorac. Cardiovasc. Surg., 39 (6): 760-766, 1960.

25 ROBERTS, W. C. - Aortic dissection: anatomy, consequences, and causes. Am. Heart J., 101 (2): 195-214. 1981.

ROSENBLUM, S. E.; RATLIFF, N. B.; SHIREY, E. K.; SEDMAK, D. D.; TAYLOR, P. C. - Pulmonary artery dissection induced by a Swan-Ganz catheter. Cleve. Clin. Q., 51 (4): 671-675, 1984
27 SHILKIN, K. B.: LOW. L. P.: CHEN, B. T. M. - Dissecting aneurysm of the pulmonary artery. J. Pathol., 98:25-29, 1969.

SCHLATMANN, T. J. M. \& BECKER, A. E. - Histologic changes in the normal aging aorta: implications for dissecting aortic aneurysm. Am. J. Cardiol., 39 (1): 13-20. 1977.

SCHLATMANN, T. J. M. \& BECKER, A. E. - Pathogenesis of dissecting aneurysm of aorta: comparative histopathologic study of significance of medial changes. Am. J. Cardiol., 39 (1): 21-26, 1977.

SCHLICHTER, J. G.; AMROMIN, G. D.; SOLWAY, A. J. L. - Dissecting aneurysms of the aorta. Arch. Int. Med., 84: (1): 558-568, 1949.

31 SHULL, W. K.; KAPADIA, S. B.; ZUBERBUHLER, J. R. - Aneurysm of the main pulmonary artery: association with patent ductus and ostium secundum defect. $\mathrm{Am}$. J. Dis. Child., 119 (6): 507-509, 1970.

32 THOMAS, G. C.: WHITELAW, D. M.: TAYLOR, H. E. Rupture of the pulmonary artery complicating rheumatic mitral stenosis. Arch. Pathol., 60: 99-103, 1955.

33 TREDAL, S. M.: CARTER, J. B.; EDWARDS, J. R. - Cystic medial necrosis of the pulmonary artery: association with pulmonary hypertension. Arch. Pathol., 97: 183-186, 1974

34 WHITAKER, W.; HEATH, D.; BROWN, J. W. - Patent ductus arteriosus with pulmonary hypertension. $\mathrm{Br}$. Heart J., 17 (2): 121-137, 1955. 